\title{
Primary Cardiac Lymphoma Case Report
}

\author{
Rios Dueñas Giovanny*, Parra Correa, Juan and Correa Ortiz J \\ Unidad de Cirugía Cardiovascular Hospital San Ignacio, Pontificia Universidad Javeriana, Colombia
}

*Corresponding author: Rios Dueñas Giovanny, Unidad de Cirugía Cardiovascular Hospital San Ignacio, Pontificia Universidad Javeriana, Bogotá, Colombia.
Received Date: July 29, 2019

Published Date: August 02, 2019

\begin{abstract}
Primary heart tumors are very rare. Reyne et al. reported a prevalence of $0.02 \%$ for heart tumors in 22 autopsies [1]. Primary heart lymphomas are even more rare, with a prevalence between heart tumors from 1 to $2 \%$ and $0.5 \%$ of all lymphomas [1,2]. We will report one case of a 69 -year-old woman with a mass in the right atrium, with hemodynamic compromise, with the histological finding of a primary heart diffuse type B lymphoma.
\end{abstract}

Keywords: Heart lymphoma; Heart tumors; Diffuse type B Lymphoma; Cardiac lymphoma; diffuse large B-cell lymphoma; Cardiac tumors

\section{Summary}

Primary tumors of the heart are quite rare. In a series of 22 autopsies conducted by Reynen et al. [1] found a prevalence for cardiac tumors of $0.02 \%$. Primary cardiac lymphoma is even rarer, with a prevalence between cardiac tumors of $1 \%$ to $2 \%$ and $0.5 \%$ of all lymphomas [1,2]. The case of a 69-year-old woman with a diagnosis of intracardiac mass will be reported, which in the histological studies of this mass evidenced compromise by primary cardiac lymphoma.

\section{Case Report}

This is a 69-year-old patient who consulted the emergency department of San Ignacio Hospital for presenting a clinical picture of functional class deterioration, progressive dyspnea at medium and low effort, associated with a palpitation sensation. In the interrogation without significant background. It is evaluated by the emergency department, which, in view of the characteristics of the clinical picture and the presence of indirect signs of heart failure, requests computerized axial tomography with a pulmonary artery protocol to rule out pulmonary embolism and thromboembolism. This study reports a moderate pericardial effusion associated with the presence of a mass that occupies $90 \%$ of the right atrium with extension to the superior and inferior vena cava. Given these findings, an assessment was requested by the cardiology service, who performed a dynamic study with a trans-esophageal echocardiogram in which the mass with soft tissue echodensity, $7.5 \times 55 \mathrm{~mm}$, with compromise of the arrival of the superior cava, was demonstrated. Endoluminal compromise of 50 to $95 \%$ in the atriocaval junction. In addition to pericardial effusion in moderate amount without echocardiographic signs of cardiac tamponade. With these findings, it is taken to the surgical medical board in which the cardiovascular surgery service considers a candidate for surgical intervention for extraction of atrial mass and pathological study of it. Medium sternotomy is performed; Dissection of fibrotic pericardium and opening thereof, pericardial effusion is identified in the images of bloody characteristics. Cardiopulmonary bypass is established; arterial cannulation of the aortic root, venous cannulation by superior vena cava and by right femoral vein due to the tumor invasion presented in the inferior vena cava. Right atrial opening, without aortic clamping. Dissection of right atrium mass is started without complete extraction, given the presence of infiltration to the posterior wall of the right atrium, superior cava, inferior cava, interatrial septum and tricuspid valve. Given the unresectability is considered auriculorrhaphy with cardiovascular prolene 4.0 in 2 planes.

Removal of aortic clamp and cardiopulmonary bypass output without complications. Aortic clamp and cardiopulmonary bypass times, $17 \mathrm{~min}$ and $32 \mathrm{~min}$ respectively. The patient is transferred to the intensive care unit for surveillance. In the results of the histological studies, diffuse large B-cell lymphoma, primary of the heart, positive for CD45, CD20 and Bcl16, cellular proliferation index (Ki67) of 70\% was identified. Negative for Bcl2, abundant CD68 positive histiocytes and CD3 positive T lymphocytes.

The pathology shows Diffuse B-cell lymphoma activated phenotype. 
- Tumor cells of high grade of malignancy positive for CD20, Bcl6 and MUM-1 with a cell proliferation index (Ki67) of 70\%. They are negative for Bcl2 and CD10.

- IAE Stadium

- Cardiac Commitment (POP resection of cardiac tumor (07/31/2017).

- $\quad$ High-intermediate risk (IPI 3).

Echocardiography:(07/24/17) Moderate eccentric hypertrophy, LVEF 60\%, AD dilated.

It goes through its postoperative period without complications. The cardiac block is reversed, and it is discharged. Later in controls the patient presented after the second click of chemotherapy, heart block. A definitive transvalent unicameral pacemaker was implanted, which is functional. The patient has been followed for 2 years. She received for treatment 6 cycles of chemotherapy, R-CHOP21 protocol x 6 cycles. Lead a life with acceptable quality. It is independent in its functions. It is in the process of recovering secondary alterations to chemotherapy Figure 1.

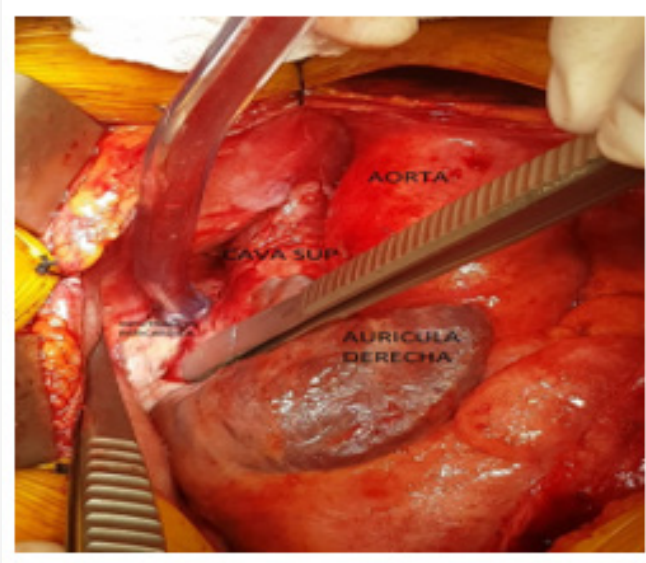

Figure 1: Tumor infiltration to pericardium

\section{Follow-up Studies have shown}

\section{8/14/2017-Macroscopic description: bone medula}

A cylindrical fragment of gray, firm, brown tissue measuring $1.5 \mathrm{~cm}$ in length and $0.3 \mathrm{~cm}$ in diameter is received. Everything is processed.

\section{Microscopic description}

The sections show a suitable cylinder of bone marrow, with a cellularity of $50 \%$, an erythroid myeloid ratio of 4 to 1 and adequate representation of the three hematopoietic lines. Myeloid line slightly expanded, maturing to polymorphonuclear, with abundant groups of central immature cells. Erythroid line with adequate nests. Megacariocytes present from 4 to 8 per field of great power, of usual morphology. The Prussian blue coloration is negative for iron deposits. The silver coloration shows a normal (+) reticular pattern. There is no evidence of hematolymphoid neoplasia.

\section{Diagnosis}

- $\quad$ Osea, Biopsy Medula

- $\quad$ Negative for Hematolinfoid Neoplasia.

- Hematopoyesis of the Three Lines

\section{Images}

\section{7/08/04 - Neck scanography with contrast medium}

No solid masses or cystic lesions were found in the different compartments of the neck. The vascular structures are normal and there are no areas of abnormal enhancement with the contrast medium. There are no cervical adenomegalies. The airway has normal caliber and the laryngeal cartilages do not present alterations. The muscle groups are normal in appearance. There is no evidence of bone injuries.

\section{Conclusion}

No signs of lymph node involvement at neck level were identified.

\section{7/08/04 - Thorax scanography with contrast medium}

Hypodense mass with lobulated margins that compromise the lateral wall of the right atrium, hypodense, with little enhancement after administration of the contrast, with signs of interatrial septum extension, has approximate greater diameters of $90 \times 49$ x $85 \mathrm{~mm}$, AP x T x L, the morphology of the other cardiac cavities is normal. Surgical changes by sternal thoracotomy approach with evidence of 2 tubes of mediastinal drainage in an adequate position without evidence of collections. There is calcified atheromatosis of the ascending aorta and the aortic arch with soft plaque of irregular margins in the anterior wall of the ascending portion. The pulmonary artery and its main branches have a usual appearance. No adenomegalies were identified in the mediastinum or in the axillae. Bilateral pleural effusion of right predominance with characteristics of free distribution. Trachea and bronchi without endoluminal lesions. Passive atelectasis underlying the pleural effusion in the right lower lobe, parenchymal bands of atelectasis in both lower lobes. There is no evidence of bone lesions or soft tissues of the chest wall.

Conclusion: Neoplastic appearance mass compromising the wall of the right atrium due to a known lymphoma. There are no signs of mediastinal lymph node involvement. Atheromatosis of the ascending aorta with soft plaques and calcified plaques described. Recent surgical changes by sternotomy approach without signs of complication.

\section{3/13/2018: PET scan of final evaluation}

without hypermetabolic lesions. Subcentimeter pulmonary nodule without increased glycolytic activity in the left lower lobe that escapes the resolution of the PET space and from which evolutionary control is recommended if deemed convenient.

11/13/2018: PET scan FDG: negative for hypermetabolic lesions 
Last evaluations by oncology in November 2018 and March 2019 where the patient evolves favobale with a functional pacemaker, no signis of tumor relapse, with recovery of hair growth, and weight. has last hemoglobin in $14.5 \mathrm{~g} / \mathrm{dl}$, courses with adequate functional class.

\section{Commentary}

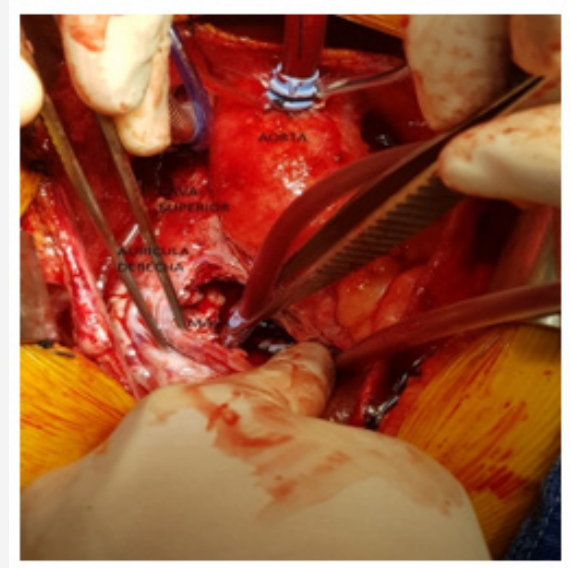

Figure 2: Exposure of the mass to the interior of the right atrium

Primary tumors of the heart are quite rare. In a series of 22 autopsies conducted by Reynen et al. [1] found a prevalence for cardiac tumors of $0.02 \%$. Primary cardiac lymphoma is even rarer with a prevalence between cardiac tumors of $1 \%$ to $2 \%$ and $0.5 \%$ of all lymphomas [1,2]. The largest case series report was conducted by Petrich et al. Where they collected 197 cases of primary cardiac lymphoma between 1947 and 2009. Reporting that this disease is more common in elderly patients, with an average age of 63 years, being more prevalent in men than in women with a radius of 2 to 1 [3-5]. The symptoms can be nonspecific, highlighting alteration in the cardiac rhythm, episodic syncope, superior vena cava syndrome, dyspnea, chills fever, sweating, weight loss and in some cases restrictive cardiomyopathy associated with pericardial effusion. Due to the non-specificity of the symptoms, the diagnosis is usually achieved with images in a hospital setting due to the worsening of the clinical condition. Transthoracic or transesophageal echocardiography is of vital importance with which the compromised thickness as well as the diameters of the affected mass and tissues, showing simultaneously if there is presence of compromise in cardiac haemodynamics Figure 2.

Primary cardiac lymphoma often involves the right heart more than the left one, it can affect both the interior of the cardiac chambers and occupy space as well as externally compressing and infiltrating nearby structures [4]. Histologically, most cases are diffuse large B-cell lymphomas. More than $80 \%$ are positive for CD20, with a high level of Ki 67 cell proliferation. An association between diffuse $\mathrm{CD} 30+\mathrm{B}$ cell lymphoma and the nucleic acid sequence of the Epstein barr virus [1] has been described. The medical treatment offered to the patient with a diagnosis of primary cardiac lymphoma is chemotherapy. The main regimen is CHOP (cyclophosphamide, doxirubicin, vincristine and prednisone), and it has been shown that associating rituximab (R-CHOP) has improved the response to chemotherapy treatment [1]. Schell et al. They used 8 cycles of R-CHOP in a 60-year-old patient with a good response and survival at 14 months [1]. Surgical treatment is reserved for patients with significant hemodynamic compromise caused by infiltration of structures such as venae cava or intracardiac extension. The prognosis of patients with primary cardiac lymphoma is poor, with a mean survival of 7 months after the initial treatment.

\section{Acknowledgement}

None.

\section{Conflict of Interest}

The authors declare that there is no conflict of interest.

\section{References}

1. Miguel C, Bestetti R (2011) Primary cardiac lymphoma. International Journal of Cardiology 149(3): 358-363.

2. Liu Y, Xu M, Fan H, Chen M, Wang J, et al. (2016) Primary cardiac lymphoma: Two rare cases. International Journal of Cardiology 203: 763-765.

3. Jonavicius K, Salcius K, Meskauskas R, Valeviciene N, Tarutis V, et al (2015) Primary cardiac lymphoma: two cases and a review of literature. Journal of Cardiothoracic Surgery 10: 138.

4. Chen S, Atlan K, Gilon D, Lotan C, Durst R, et al. (2015) Lymphoma of the Right Atrium and Ventricle. IMAJ 17(11): 712-713.

5. Chim CS, Chan AC, Kyong YL, Liang R (1997) Primary cardiac lymphoma Am J Hematol 54: 79-83. 